\title{
Efetividade de uma intervenção nutricional associada à prática de atividade física
}

\author{
Effectiveness of a nutritional intervention \\ associated with physical activity practice
}

\author{
Clesiane Honorato Machado ${ }^{1}$, Ariene Silva do Carmo ${ }^{1}$, Paula Martins Horta², \\ Aline Cristine Souza Lopes ${ }^{3}$, Luana Caroline dos Santos ${ }^{3}$
}

\begin{abstract}
Resumo
A transição nutricional no Brasil suscita a criação de ambientes e contextos promotores de modos mais saudáveis de vida. Objetivou-se verificar a efetividade de uma intervenção nutricional associada à prática de atividade física entre usuários de um Serviço de Promoção da Saúde de Belo Horizonte (MG). A intervenção foi realizada com usuários adultos eutróficos ou com sobrepeso e idosos eutróficos e teve duração de 6 meses. Avaliaram-se, antes e após esse período, condição sociodemográfica e econômica, perfil de saúde, consumo alimentar (hábitos alimentares e Questionário de Frequência Alimentar) e antropometria. Participaram do estudo 20 usuários, sendo 95\% mulheres, 75\% adultos, com 52,0 12,9 anos. Avaliação inicial evidenciou a presença de hábitos alimentares inadequados e $30 \%$ de excesso de peso entre os participantes. Após a intervenção nutricional não houve alteração no que concerne aos hábitos alimentares, porém verificou-se maior adequação no consumo de embutidos ( $p=0,016)$. Além disso, houve redução do peso corporal $(62,6 \pm 8,2$ versus $61,7 \pm 8,8 ; p=0,032)$, circunferência de cintura $(82,5 \pm 8,2$ versus $78,9 \pm 6,2 ; p=0,002)$ e relação cintura/quadril $(0,82 \pm 0,07$ versus $0,79 \pm 0,05 ; p=0,016)$. A intervenção foi efetiva para mudanças antropométricas e para redução do consumo de embutidos, destacando-se a importância desses serviços para a promoção da saúde da população.
\end{abstract}

Palavras-chave: atenção primária à saúde; consumo de alimentos; hábitos alimentares; promoção da saúde.

\begin{abstract}
The nutritional transition in Brazil demands the development of contexts and environments of healthy lifestyles promotion. This study aimed to evaluate the effectiveness of a nutritional intervention associated with physical activity practice among individuals from a Health Promotion Service of Belo Horizonte (MG). The intervention was carried out in six months with normal weight or overweight adults and normal weight elderly. Socio-demographic and economic condition was evaluated before and after this period in addition to health profile, food consumption (eating habits and food frequency questionnaire) and anthropometry. The sample included 20 subjects, 95\% women, 75\% adults, 52.0 \pm 12.9 years. Initially, evaluation revealed occurrence of inadequate eating habits and $30 \%$ of overweight among participants. After the nutritional intervention period, there were no differences regarding eating habits, but an increase in the adequacy of processed meats consumption was verified $(p=0.016)$. Furthermore, a reduction in body weight $(62.6 \pm 8.2$ versus $61.7 \pm 8.8, p=0.032)$, waist circumference $(82.5 \pm 8.2$ versus $78.9 \pm 6.2 ; p=0.002)$ and waist-to-hip ratio $(0.82 \pm 0.07$ versus $0.79 \pm 0.05, p=0.016)$. The intervention was effective for anthropometry changes and reduction in processed meats consumption, highlighting the importance of these services for the population health promotion.
\end{abstract}

Keywords: primary health care; food consumption; food habits; health promotion.

Trabalho realizado na Universidade Federal de Minas Gerais (UFMG) - Belo Horizonte (MG), Brasil.

${ }^{1}$ Nutricionista; Mestranda do programa de Pós Graduação em Enfermagem e Saúde na linha de pesquisa Prevenção e Controle de Agravos à Saúde da UFMG Belo Horizonte (MG), Brasil.

${ }^{2}$ Nutricionista; Mestre em Saúde e Enfermagem pela UFMG e Doutoranda em Ciências da Saúde na linha de pesquisa Saúde da Criança e do Adolescente da UFMG - Belo Horizonte (MG), Brasil.

${ }^{3}$ Doutora em Saúde Pública; Professora Adjunta do Curso de Nutrição e da Pós-Graduação em Enfermagem e Saúde da UFMG - Belo Horizonte (MG), Brasil. Endereço para correspondência: Clesiane Honorato Machado - Avenida Professor Alfredo Balena, 190, sala 428 - CEP: 30130-100 - Belo Horizonte (MG), Brasil E-mail: clesianehonorato@gmail.com

Fonte de financiamento: FAPEMIG - Fundação de Amparo à Pesquisa do estado de Minas Gerais.

Conflito de interesse: nada a declarar. 


\section{INTRODUÇÃO}

O Brasil, nos últimos anos, vem enfrentando um aumento na prevalência das doenças e agravos não transmissíveis (DANT), a exemplo do diabetes mellitus (DM), hipertensão arterial sistêmica (HAS) e dislipidemias, que se relacionam à elevada ocorrência de incapacidades, mortes e utilização dos serviços de saúde ${ }^{1}$.

Resultados da pesquisa nacional Vigilância de Fatores de Risco e Proteção para Doenças Crônicas por Inquérito Telefônico apontam que a prevalência autorreferida de HAS e DM na população brasileira é de 22,7 e 5,6\%, respectivamente 2 .

Esse quadro suscita a adoção de estratégias integradas e sustentáveis de controle dessas doenças, que atuem sobre os seus principais fatores de risco, destacando-se tabagismo, inatividade física e alimentação inadequada ${ }^{3}$. Para tal, sugerese uma abordagem fundamentada na promoção à saúde e na constituição de ambientes e contextos promotores de modos mais saudáveis de vida ${ }^{4}$.

Nesse sentido, foram propostos, no Brasil, espaços de promoção à saúde que incentivam a prática de atividades no lazer e de exercícios físicos, além da alimentação saudável. Alguns exemplos incluem o programa Agita São Paulo, o CuritibAtiva e as Academias da Cidade de Recife (PE) ${ }^{5-7}$. O programa Agita São Paulo foi o primeiro a ser implantado, em 1996, com o objetivo de mudar o comportamento geral da população quanto à prática de atividade física a partir de mensagens sobre o benefício dessa prática à saúde além de atividades coordenadas e intervenções 5 .

Em Belo Horizonte (MG) também estão implantadas as Academias da Cidade desde 2006, com o objetivo de viabilizar intervenção nutricional e prática de atividade física gratuita a indivíduos residentes em regiões de vulnerabilidade social ${ }^{8}$.

De forma similar, o Ministério da Saúde propôs a implantação em nível nacional das Academias da Saúde em 2011. Essas têm como objetivo principal contribuir para a promoção da saúde da população a partir da implantação de polos com infraestrutura, equipamentos e quadro de pessoal qualificado para a orientação de práticas corporais e atividade física e de lazer e modos de vida saudáveis?.

Considerando a importância da promoção da saúde para a prevenção e controle de DANT, o presente estudo objetivou verificar a efetividade de uma intervenção nutricional associada à prática de atividade física sobre o perfil alimentar e antropométrico de usuários de uma Academia da Cidade de Belo Horizonte (MG).

\section{METODOLOGIA}

Trata-se de um estudo de intervenção não controlada, com duração de seis meses, desenvolvido com usuários de um Serviço de Promoção à Saúde, denominado Academia da Cidade, de Belo Horizonte (MG). Tal serviço consiste em uma das estratégias do Projeto BH-Mais-Saudável para promoção da saúde no município e oferece gratuitamente a prática regular de exercícios físicos e orientação nutricional, individual e coletiva, aos participantes ${ }^{8}$.

O estudo incluiu todos os adultos eutróficos ou com sobrepeso e os idosos eutróficos ingressos no serviço por demanda espontânea ou encaminhamento do profissional de saúde para a prática de exercícios físicos no turno matutino. Os critérios para atendimento nutricional foram definidos pelo Grupo Intersetorial de Promoção à Saúde, sendo que os demais usuários não contemplados por esses critérios foram encaminhados para o acompanhamento nutricional pelo Núcleo de Apoio à Saúde da Família (NASF) nas Unidades Básicas de Saúde (UBS) de referênciå.

Considerando a elevada rotatividade do serviço, definiuse como critério de exclusão à amostra não ter completado seis meses de atendimento nutricional, ter-se desvinculado da Academia da Cidade nesse período, ter reavaliação nutricional realizada em período superior a sete meses, não ter realizado pelo menos dois retornos para consulta nutricional nesse intervalo ou ter-se recusado em participar do estudo.

Os atendimentos nutricionais foram realizados por acadêmicos de Nutrição da Universidade Federal de Minas Gerais (UFMG) treinados e supervisionados com duração de, aproximadamente, uma hora. Os dados foram obtidos com o uso de um questionário estruturado aplicado na primeira consulta e após seis meses de intervenção nutricional, abrangendo informações sociodemográficas e econômicas (idade, sexo, escolaridade e renda per capita), autorrelato de morbidades (HAS, DM e dislipidemias), prática de atividade física (horas semanais), consumo alimentar (hábitos alimentares e Questionário de Frequência Alimentar (QFA)) e antropometria (índice de massa corporal (IMC), circunferência de cintura (CC) e relação cintura/quadril (RCQ) $)^{10}$.

No que concerne aos hábitos alimentares, foram avaliados o consumo de gordura aparente da carne e da pele de frango, o hábito de se alimentar extemporaneamente ("beliscar" alimentos entre as refeições) e qualidade da mastigação dos alimentos.

O QFA, por sua vez, foi do tipo qualitativo, e contemplou a frequência de consumo de frutas, hortaliças, leite e seus derivados, embutidos, biscoito recheado, doces em geral, frituras, salgados, salgadinhos do tipo chips e sanduíches, suco em pó e refrigerante ${ }^{10}$. O consumo desses alimentos foi classificado em adequado e não adequado, conforme critérios adotados por Andrade et al. ${ }^{11}$. 
Considerando a avaliação antropométrica, o IMC (peso $(\mathrm{kg}) /$ altura $(\mathrm{m})^{2}$ ) foi classificado de maneira diferenciada para adultos ${ }^{12}$ e idosos ${ }^{13}$. Para a classificação da CC e da RCQ (CC/circunferência de quadril (CQ)) foram seguidos os critérios propostos pela Organização Mundial da Saúde (OMS ${ }^{14}$.

A partir da aplicação do questionário de identificação do perfil nutricional, os usuários foram acompanhados mensalmente e ao final de cada atendimento foram fornecidas orientações nutricionais, baseadas nas diretrizes do Guia Alimentar para a População Brasileira e demais manuais do Ministério da Saúde ${ }^{4,15-17}$.

Além da intervenção nutricional, os usuários foram acompanhados por uma Equipe de Educação Física composta por dois profissionais e dois estagiários para a realização de exercícios físicos. Os mesmos ocorreram três vezes por semana, sendo cada aula com duração de uma hora, incluindo exercícios aeróbios e anaeróbios ${ }^{8}$.

Foram utilizados os programas Epi Info 6.04 para a construção do banco de dados e o Statistical Package for the Social Sciences (SPSS) para Windows versão 19.0 para as análises estatísticas. Realizou-se análise descritiva dos dados, com cálculo das frequências, medidas de tendência central e de dispersão e aplicação do teste de normalidade de Kolmogorov-Smirnov para avaliação da aderência das variáveis quantitativas à distribuição normal. Ademais, aplicaram-se os testes para amostras dependentes $t$ de Student Pareado e McNemar.

Os resultados foram apresentados sob a forma de média e desvio padrão para as variáveis com distribuição normal e mediana e intervalo de confiança de 95\% (IC95\%) para as demais. O nível de significância adotado foi de $5 \%(\mathrm{p}<0,05)$.

O estudo está inserido no projeto "Desenvolvimento e avaliação de intervenções nutricionais realizadas nas academias da cidade pertencentes ao Projeto BH Saúde Belo Horizonte, MG", aprovado pelo Comitê de Ética em Pesquisa da Secretaria Municipal de Saúde de Belo Horizonte (Protocolo 087/2007) e da UFMG (COEP ETIC 103/07).

\section{RESULTADOS}

Foram entrevistados 316 usuários no período de realização do estudo, sendo que 151 (47,8\%) eram contemplados pelos critérios de acompanhamento nutricional ${ }^{8}$. A partir desse total, foram incluídos ao estudo 20 usuários, tendo em vista os citados critérios de exclusão à amostra (Figura 1).

Desses, 95\% ( $n=19)$ eram do sexo feminino e $75 \%(n=15)$ adultos, com média de 52,0 $\pm 12,9$ anos. Verificou-se média de $8,3 \pm 4,2$ anos de estudo, renda per capita de $\mathrm{R} \$ 594,6 \pm 473,7$ e mediana de 3 horas (IC95\% 1,5-6,0) semanais de prática de atividade física. Em relação à presença de morbidades, 15\% $(n=3)$ dos indivíduos relataram possuir HAS e dislipidemias, e ninguém relatou a presença de DM.

Considerando os hábitos alimentares, antes da intervenção nutricional verificou-se que $40 \%$ dos usuários referiram mastigação inadequada dos alimentos e 55\% apresentavam o hábito de "beliscar" entre as refeições (Tabela 1). Após a intervenção não foram verificadas alterações com significância estatística nestes e demais hábitos alimentares (Tabela 1 ).

A análise do QFA evidenciou 75\% e 60\% de inadequação do consumo de hortaliças e frutas, respectivamente, no momento de ingresso no serviço. Após a intervenção nutricional, houve maior adequação no consumo de embutidos $(\mathrm{p}=0,016)$ (Tabela 2).

Quanto ao estado nutricional, 70\% da amostra era eutrófica ao início do estudo. De acordo com a classificação da CC, $25 \%$ encontrava-se com risco elevado e $25 \%$ com risco muito elevado de complicações associadas à obesidade, e $40 \%$ apresentavam risco para o desenvolvimento de doenças cardiovasculares de acordo com a RCQ. Com a intervenção, verificou-se redução do peso corporal $(62,6 \pm 8,2$ versus $61,7 \pm 8,8 ; \mathrm{p}=0,032), \mathrm{CC}(82,5 \pm 8,2$ versus $78,9 \pm 6,2 ; \mathrm{p}=0,002)$ e $\operatorname{RCQ}(0,82 \pm 0,07$ versus $0,79 \pm 0,05 ; \mathrm{p}=0,016)$. Ademais, não foi identificada diferença estatisticamente significativa do IMC e da classificação desses índices antropométricos após a intervenção $(p>0,05)$.

Indivíduos entrevistados: $\mathrm{n}=316$

Excluídos indivíduos que não atendiam aos critérios para acompanhamento nutricional mensal no local $(n=165)$

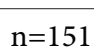

Excluídos indivíduos que não completaram seis meses de atendimento nutricional $(\mathrm{n}=84)$

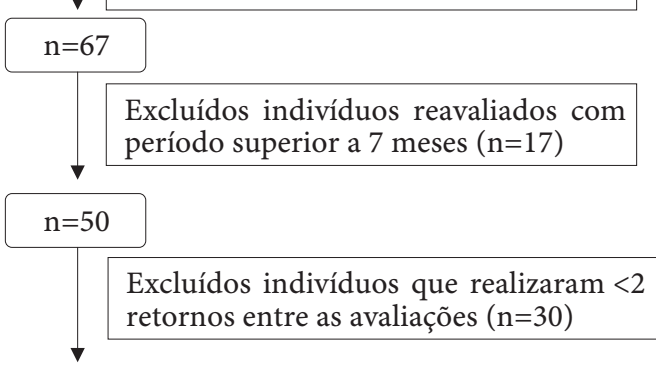

Amostra final: $\mathrm{n}=20$

Figura 1. Definição da amostra do estudo. Serviço de Promoção da Saúde de Belo Horizonte (MG), 2012 
Tabela 1. Hábitos alimentares antes e após intervenção nutricional de usuários de um Serviço de Promoção da Saúde de Belo Horizonte (MG), 2012

\begin{tabular}{|c|c|c|c|c|c|}
\hline \multirow{2}{*}{ Variáveis } & \multicolumn{2}{|c|}{ Pré-intervenção (\%) } & \multicolumn{2}{|c|}{ Pós-intervenção (\%) } & \multirow[t]{2}{*}{ Valor $\mathrm{p}^{x}$} \\
\hline & Sim & Não & Sim & Não & \\
\hline Consome gordura aparente da carne & 31,2 & 68,8 & 0,0 & 100,0 & 0,910 \\
\hline Consome pele de frango & 10,0 & 90,0 & 7,7 & 92,3 & 1,000 \\
\hline Alimenta extemporaneamente & 45,0 & 55,0 & 30,0 & 70,0 & 0,250 \\
\hline Mastiga bem os alimentos & 60,0 & 40,0 & 60,0 & 40,0 & 1,000 \\
\hline
\end{tabular}

*Teste MC Nemar

Tabela 2. Consumo alimentar antes e após intervenção nutricional de usuários de um Serviço de Promoção da Saúde de Belo Horizonte (MG), 2012

\begin{tabular}{|c|c|c|c|c|c|}
\hline \multirow{2}{*}{ Alimento } & \multicolumn{2}{|c|}{ Pré-intervenção (\%) } & \multicolumn{2}{|c|}{ Pós-intervenção (\%) } & \multirow{2}{*}{ Valor $\mathrm{p}^{\times}$} \\
\hline & Adequado & Inadequado & Adequado & Inadequado & \\
\hline Frutas & 40,0 & 60,0 & 35,0 & 65,0 & 1,000 \\
\hline Hortaliças & 25,0 & 75,0 & 35,0 & 65,0 & 0,687 \\
\hline Leite e derivados & 45,0 & 55,0 & 30,0 & 70,0 & 0,375 \\
\hline Embutidos & 55,0 & 45,0 & 90,0 & 10,0 & 0,016 \\
\hline Biscoitos recheados & 95,0 & 5,0 & 95,0 & 5,0 & 1,000 \\
\hline Doces em geral & 75,0 & 25,0 & 85,0 & 15,0 & 0,687 \\
\hline Frituras & 70,0 & 30,0 & 85,0 & 15,0 & 0,453 \\
\hline Salgados, chips e sanduíches & 95,0 & 5,0 & 95,0 & 5,0 & 1,000 \\
\hline Sucos em pó & 85,0 & 15,0 & 90,0 & 10,0 & 1,000 \\
\hline Refrigerantes & 95,0 & 5,0 & 95,0 & 5,0 & 1,000 \\
\hline
\end{tabular}

*Teste MC Nemar

\section{DISCUSSÃO}

A intervenção nutricional aliada à atividade física resultou em melhor adequação no consumo de embutidos e mudanças positivas na antropometria.

A redução no consumo de embutidos é benéfica à saúde, uma vez que esse alimento contém grandes quantidades de sal e gordura saturada ${ }^{18,19} \mathrm{e}$ o consumo excessivo destes componentes dietéticos está associado ao maior risco de doenças cardiovasculares ${ }^{20,21}$.

Em relação às mudanças dietéticas, observou-se também um aumento percentual não significativo de alguns hábitos alimentares e da adequação do consumo de alimentos avaliados pelo QFA. Apesar de tais mudanças não serem significantes estatisticamente, parece que pequenas modificações do padrão alimentar, mesmo não alterando em curto prazo o estado de saúde, podem ser alcançadas com modelos de aconselhamento nutricional ${ }^{22}$. Ademais, destaca-se que a alta prevalência de hábitos alimentares inadequados verificados no momento pré-intervenção denota a importância da promoção de práticas alimentares saudáveis nesse serviço.

Quanto à redução de peso e da CC, tais achados corroboram outros autores que utilizaram a promoção de alimentação saudável em conjunto com o incentivo à prática regular de atividade física em suas intervenções ${ }^{5,23,24}$.

Esses resultados podem acarretar a melhoria significativa da saúde dos usuários, tais como prevenção do risco de obesidade e de desenvolvimento de doenças, além do melhor controle das comorbidades associadas, com consequente melhoria da qualidade e expectativa de vida ${ }^{24}$.

Monteiro, Riether e Burini, em estudo de intervenção de 40 semanas conduzido com mulheres em climatério $(n=30)$, também verificou redução de peso e da CC entre as participantes, sendo esses valores maiores para o grupo que sofreu intervenção nutricional aliada à prática de atividade física em comparação ao grupo que participou somente da intervenção nutricional ${ }^{25}$. Já Ferreira et al. ${ }^{26}$ avaliaram 64 mulheres, com idade entre 50 e 72 anos, divididas em 4 grupos 
de acordo com o tipo de intervenção recebida (orientação nutricional; orientação nutricional e atividade física; orientação sobre atividade física e nenhum tipo de orientação) e não encontraram redução na antropometria e adiposidade corporal após 12 semanas de intervenção. Entretanto, segundo o mesmo autor, mesmo não encontrando mudanças nas variáveis antropométricas analisadas, não se pode eliminar a hipótese de que orientações nutricionais e de atividade física resultem em mudanças voluntárias nos hábitos alimentares e que essas mudanças resultem em alterações positivas na composição corporal ${ }^{26}$.

Considera-se, como limitação do presente estudo, a ausência de grupo controle e o limitado tamanho amostral. Porém, os resultados positivos alcançados neste trabalho evidenciam a importância de serviços, como as Academias da Cidade, que aliam a prática regular de exercício físico à orientação nutricional para a promoção e recuperação da saúde dos usuários.

Sugere-se a continuidade da intervenção nutricional nesse tipo de serviço, a fim de consolidar as mudanças em longo prazo, bem como a avaliação da intervenção em um período maior.

\section{CONCLUSÃO}

A intervenção foi efetiva para mudanças antropométricas e redução do consumo de embutidos, destacando-se a importância de tais serviços na promoção da melhor qualidade de vida e redução de riscos de desenvolvimento de doenças crônicas.

\section{REFERÊNCIAS}

1. Brasil. Ministério da Saúde. Secretaria de Vigilância em Saúde. Secretaria de Atenção à Saúde. Diretrizes e recomendações para o cuidado integral de doenças crônicas não-transmissíveis: promoção da saúde, vigilância, prevenção e assistência. Brasília: Ministério da Saúde; 2008.

2. Brasil. Ministério da Saúde. VIGITEL BRASIL 2011 - Vigilância de fatores de risco e proteção para doenças crônicas por inquérito telefônico: estimativas sobre frequência e distribuição sociodemográfica de fatores de risco e proteção para doenças crônicas nas capitais dos 26 estados brasileiros e no distrito federal em 2011. Brasília; 2012.

3. Barros RMM, Fontinele RSS, Seyffarth AS. Intervenção nutricional em grupo de moradores atendidos pelo Programa Família Saudável. Comun Ciênc Saúde. 2007;18(3):207-14.

4. Brasil. Ministério da Saúde. Secretaria de Atenção à Saúde. Departamento de Atenção Básica. Guia alimentar para a população brasileira: promovendo a alimentação saudável. 2008.

5. Matsudo SM, Matsudo VKR, Andrade DR, Araújo TL, Pratt M. Evaluation of a physical activity promotion program: The example of Agita São Paulo. Eval Prog Planning. 2006;29(3):301-11.

6. Hallal PC, Reis RS, Hino AAF, Santos MS, Grande D, Krempel MC et al Avaliação de programas comunitários de promoção da atividade física: o caso de Curitiba, Paraná. Rev Bras Ativ Fís Saúde. 2009; 14(2):104-14.

7. Hallal PC, Tenório MCM, Tassitano RM, Reis RS, Carvalho YM, Cruz DKA, et al. Avaliação do programa de promoção da atividade física Academia da Cidade de Recife, Pernambuco, Brasil: percepções de usuários e não-usuários. Cad Saúde Pública. 2010;26(1):70-8.

8. Dias MAS, Lopes ACS, Bicalho K, Mourão M, Alves MN, Evangelista PA, et al. Promoção à saúde e articulação intersetorial. In: Magalhães Júnior HM (Org). Desafios e inovações da gestão do SUS em Belo Horizonte: a experiência de 2003 a 2008. Belo Horizonte: Mazza Edições. 2010; v. 1, p. 63-124.

9. Brasil. Ministério da Saúde. Portaria 719 [Internet]. 2011 [citado em 16 fev. 2012]. Disponível em: http://portal.saude.gov.br/portal/arquivos/ pdf/portaria_academia_saude_719.pdf
10. Lopes ACS, Ferreira AD, Santos LC. Atendimento nutricional na atenção primária à saúde: proposição de protocolos. Nutr Pauta. 2010;18(101):40-4.

11. Andrade KA, Toledo MTT, Lopes MS, Carmo GES, Lopes ACS. Aconselhamento sobre modos saudáveis de vida na atenção primária e práticas alimentares dos usuários. Rev Esc Enferm USP. 2012;46(5):1117-24.

12. WHO - World Health Organization. Obesity: Preventing and managing the global epidemic - Report of a WHO consultation on obesity. Geneva; 1998.

13. NSI - Nutrition Screening Initiative. Nutrition interventions manual for professionals caring for older americans: project of the American Academy of Family Physicians. The American Dietetic Association and National Council on Aging Washington (DC): 1994.

14. WHO - World Health Organization. Waist circumference and waist-hip ratio. Report of a WHO expert consultation. Geneva; 2008.

15. Brasil. Ministério da Saúde. Secretaria de Atenção à Saúde. Departamento de Atenção Básica. Diabetes Mellitus. Caderno de Atenção Básica, n.16. Brasília: Ministério da Saúde; 2006.

16. Brasil. Ministério da Saúde. Secretaria de Atenção à Saúde. Departamento de Atenção Básica. Hipertensão arterial sistêmica. Caderno de Atenção Básica, n.15. Brasília: Ministério da Saúde; 2006.

17. Brasil. Ministério da Saúde. Secretaria de Atenção à Saúde. Departamento de Atenção Básica. Obesidade. Caderno de Atenção Básica, n.12. Brasília: Ministério da Saúde; 2006. 108 p.

18. Nakasato M. Sal e hipertensão arterial. Rev Bras Hipertens. 2004;11(2):95-7.

19. Muniz LC, Schneider BC, Silva ICM, Matijasevich A, Santos IS. Fatores de risco comportamentais acumulados para doenças cardiovasculares no sul do Brasil. Rev Saúde Pública. 2012;46(3):534-42.

20. Ruivo GF, Heimann JC. Revisão: O efeito do sal na resistência à insulina evidências clínicas e experimentais. J Bras Nefrol. 2003;25(1):34-40.

21. Woodside JV, Kromhout D. Fatty acids and CHD. Proc Nutr Soc. 2005;64(4):554-64. 
22. Guimarães NG, Dutra ES, Ito $M K$, Carvalho KMB. Adesão a um programa de aconselhamento nutricional para adultos com excesso de peso e comorbidades. Rev Nutr [online]. 2010;23(3):323-33.

23. Costa PRF, Assis AMO, Silva MCM, Santana MLP, Dias JC, Pinheiro SMC, et al Mudança nos parâmetros antropométricos: a influência de um programa de intervenção nutricional e exercício físico em mulheres adultas. Cad Saúde Pública. 2009;25(8):1763-73.

24. Goldberg Y, Boaz M, Matas Z, Goldberg I, Shargorodsky M. Weight loss induced by nutritional and exercise intervention decreases arterial stiffness in obese subjects. Clin Nutr. 2009;28(1):21-5.
25. Monteiro RCA, Riether PTA, Burini RC. Efeito de um programa misto de intervenção nutricional e exercício físico sobre a composição corporal e os hábitos alimentares de mulheres obesas em climatério. Rev Nutr. 2004;17(4):479-89.

26. Ferreira M, Matsudo S, Matsudo V, Braggion G. Efeitos de um programa de orientação de atividade física e nutricional sobre a ingestão alimentar e composição corporal de mulheres fisicamente ativas de 50 a 72 anos. Rev Bras Ciênc Mov. 2003;11(1):35-40.

Recebido em: 14/03/2013 Aprovado em: 06/06/2013 